\title{
FERIMENTOS CERVICAIS: ANÁLISE RETROSPECTIVA DE 191 CASOS
}

NECK INJURIES: RETROSPECTIVEANALYSIS OF 191

\author{
Luiz Carlos Von Bahten, TCBC-PR ${ }^{1}$ \\ João Ricardo Duda ${ }^{2}$ \\ Patrícia Danielle Schultz Zanatta ${ }^{2}$ \\ Andrei Leite de Morais ${ }^{3}$ \\ Fábio Silveira ${ }^{4}$ \\ Márcia Olandoski ${ }^{5}$
}

\begin{abstract}
RESUMO: Objetivos: Analisar a epidemiologia e a conduta nos ferimentos cervicais. Método: Foram analisados 487.128 prontuários de pacientes que ingressaram no Serviço de Emergência do Hospital Universitário Cajuru no período de 01/1996 a 06/2001. Destes, selecionaram-se 378 pacientes com ferimentos cervicais. Foram excluídos 153 que apresentavam lesões associadas e 14 por óbito no atendimento inicial. O estudo foi feito, assim, em 191 pacientes com lesões cervicais exclusivas. Avaliou-se a localização da ferida, o mecanismo de trauma, o comprometimento do platisma, sinais e sintomas, a hora de admissão e a conduta empregada. Resultados: Cento e sessenta e quatro (86\%) pacientes eram masculinos. A média de idade foi de 28 anos (1072). Noventa (47\%) ferimentos foram por arma de fogo (FAF) e $88(46 \%)$ por arma branca (FAB). O principal horário de admissão foi entre 20 e 04 horas. Quanto à localização, 53\% das lesões foram à esquerda, 45\% à direita e $2 \%$ medianos; $36 \%$ em zona I, 55\% em zona II e 9\% em zona III. Em 101 o ferimento penetrou o platisma: cinqüenta e um (50\%) apresentaram sinais e sintomas clínicos e receberam conduta operatória. As lesões vasculares foram as mais encontradas (20). Houve 24 (47\%) cervicotomias não-terapêuticas. O tratamento conservador foi empregado em 41 (45\%) casos de acordo com os exames físico e complementares. Conclusões: Homens jovens são mais acometidos quanto aos ferimentos cervicais. Estes ocorrem mais freqüentemente na zona II, e a incidência dos FAF e FAB foi equivalente. É adequado um manejo mais seletivo em relação aos ferimentos cervicais, devendo o manejo da zona II adequar-se à disposição de recursos dos serviços de trauma.
\end{abstract}

Descritores: Lesões do pescoço; Epidemiologia; Análise; Condutas terapêuticas.

\section{INTRODUÇÃO}

São muitas as controvérsias e dificuldades em torno da avaliação e tratamento dos ferimentos penetrantes de pescoço. Anatomicamente observa-se uma diversidade das estruturas vasculares, respiratórias, digestivas e nervosas que se dispõem de maneira muito próxima e particular no pescoço. Existe atualmente um grande debate em torno da conduta a ser adotada em tais lesões e o manejo operatório vem sendo substituído por uma abordagem mais seletiva e conservadora. Questionamentos têm sido feitos relacionando-

1. Prof. Dr. Titular da Disciplina de Cirurgia da PUC PR e Prof. Adjunto da Disciplina de Cirurgia do Trauma UFPR.

2. Alunos do Curso de Medicina da PUCPR.

3. Médico Residente de Neurocirurgia do HNSG - Curitiba.

4. Médico Residente de Clínica Cirúrgica da Aliança Saúde Hospitais Cajuru - Santa Casa de Curitiba.

5. Prof ${ }^{\text {a }}$ de Bioestatística do Centro de Ciências Biológicas e da Saúde da PUCPR.

Recebido em 06/11/2002

Aceito para publicação em 28/07/2003

Trabalho realizado no Serviço de Cirurgia Geral do Hospital Universitário Cajuru da PUCPR, Curitiba-Paraná-Brasil. 
se mecanismo de lesão e a sua conduta, qual o melhor exame diagnóstico na conduta não-operatória, quando optar pelo tratamento cirúrgico e qual a melhor técnica para o reparo cirúrgico das diferentes lesões encontradas.

O pescoço pode ser dividido anatomicamente em zonas (I, II e III) e trígonos (anterior e posterior). A zona I está compreendida entre as clavículas e a cartilagem cricóide. A zona II entre a cartilagem cricóide e o ângulo da mandíbula e a zona III, estende-se deste ângulo até a mastóide. ${ }^{1} \mathrm{O}$ trígono anterior é limitado pela borda inferior da mandíbula, o músculo esternocleidomastoídeo e a linha mediana anterior do pescoço. Em relação ao trígono posterior os limites são: a face superior da clavícula, o músculo trapézio e o músculo esternocleidomastoídeo. ${ }^{2}$ (Figura1)
Os ferimentos de pescoço podem ser superficiais ou penetrantes. São considerados penetrantes os que ultrapassam o músculo platisma. ${ }^{3} \mathrm{~A}$ avaliação e o controle iniciais devem ser baseados no protocolo do Advanced Trauma Life Support (ATLS). ${ }^{4}$

Quanto ao sexo dos pacientes vítimas destas lesões, há predominância do sexo masculino, com aproximadamente $90 \%$ dos doentes..$^{2,3,5-8}$ A faixa etária mais acometida está entre 24 e 34 anos. .,5-10 $^{3}$

Em relação ao local dos ferimentos, a zona II, com 47 a $82 \%$ dos ferimentos, é a área onde mais se observam as lesões, seguida das zonas I ( 5 a $31 \%$ ) e III (1 a 30\%) ${ }^{5-9,11-14}$. As lesões vasculares são as lesões mais freqüentes nos traumatismos cervicais penetrantes $(21 \text { a } 27 \%)^{15-17}$. A seguir, observam-se as lesões neurológicas $(16 \%)^{15}$ e as do trato aerodigestório (6 a 10\%). ${ }^{15,18}$

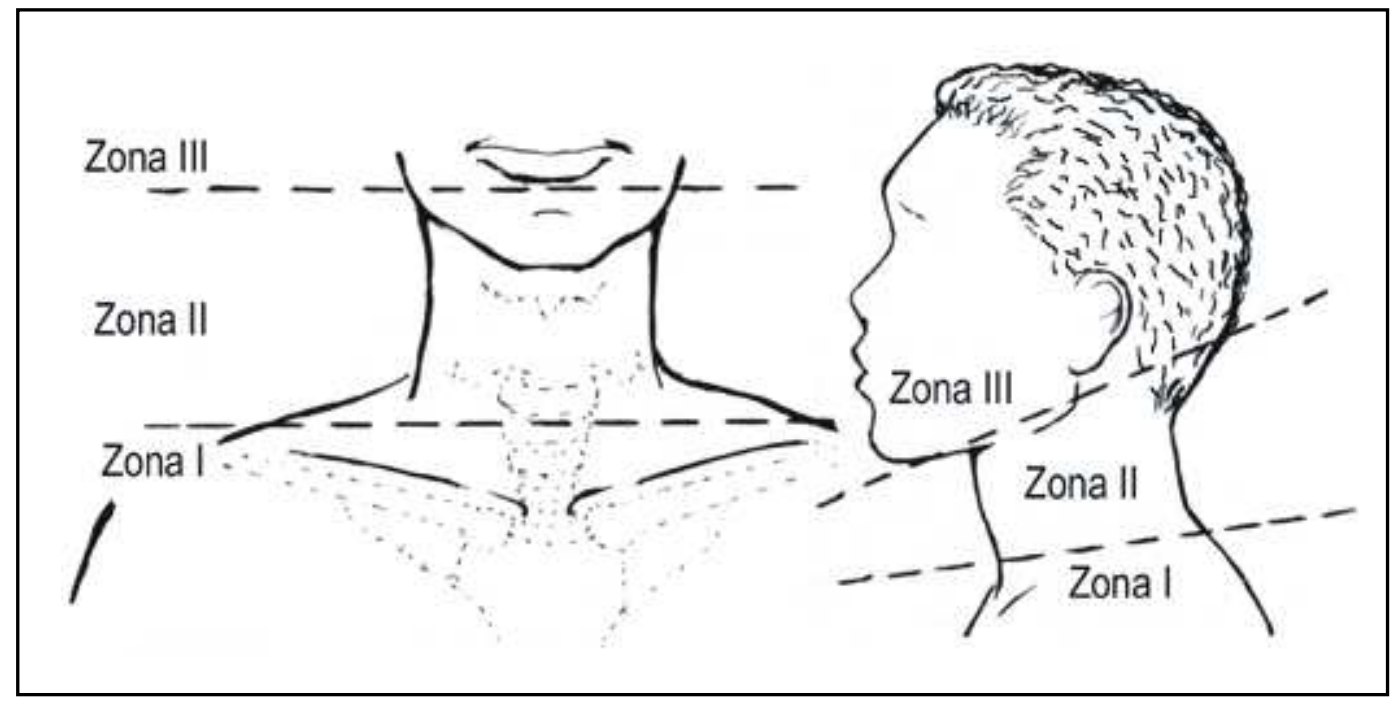

Figura 1 - Zonas Anatômicas do Pescoço.

(Mattox KL, Feliciano DV, Moore EE - Trauma, 4a. ed. p. 438. United States of America - McGraw-Hill, 2000.)

Existem ainda controvérsias entre os autores quanto ao tratamento operatório ou conservador das lesões penetrantes de pescoço. Alguns optam pela exploração mandatória, outros apenas pelo exame físico e outros selecionam a terapêutica para seus pacientes de acordo com a zona acometida, explorando de imediato ou utilizando-se dos exames complementares. Esta preocupação se deve à tentativa de se evitar operações não terapêuticas, que ocorrem em larga escala quando do uso de condutas cirúrgicas mandatórias ${ }^{11,19-23}$. O grande debate ainda se refere aos ferimentos da zona II. Segundo Asensio ${ }^{24}$, ambos os tratamentos se equivalem, pois uma exploração indispensável é segura e aceitável, enquanto a abordagem seletiva com angiografia nos assintomáticos também é muito eficaz.

Em virtude da complexidade no manejo de pacientes com trauma cervical e de sua importância social e epidemiológica, analisamos estatisticamente o mecanismo de trauma, sua localização, os eventuais sinais e sintomas clínicos, bem como da conduta adotada nestes pacientes. 


\section{MÉTODO}

Foram avaliados 487.128 prontuários de diferentes especialidades médicas de pacientes que ingressaram no serviço de emergência do Hospital Universitário Cajuru no período de janeiro de 1996 a julho de 2001. O Hospital Cajuru é referência em emergências na cidade de Curitiba, com 2 milhões de habitantes.

Destes prontuários, selecionaram-se 378 pacientes que apresentaram ferimentos de pescoço. Foram excluídos 153 pacientes por se apresentarem com lesões associadas e 14 pacientes por óbito dentro do atendimento inicial (comprometimento de via aérea e/ou choque hipovolêmico (grau IV).

Desta forma, selecionaram-se os 191 pacientes restantes que apresentaram apenas lesões cervicais. Para análise dos prontuários destes pacientes utilizou-se o Protocolo de Feridas Penetrantes de Pescoço do Colégio Americano de Cirurgiões (Clínicas Cirúrgicas da América do Norte, 1996) ${ }^{16}$.

Determinaram-se o sexo e a faixa etária média dos pacientes.

$\mathrm{Na}$ avaliação da localização da ferida observam-se os hemídios (direito ou esquerdo), trígonos (anterior e posterior) e zonas (I, II ou III). Avaliou-se o mecanismo de trauma (arma de fogo ou arma branca), o comprometimento do músculo platisma, sinais e sintomas (sangramento ativo, enfisema subcutâneo, hemoptise, escape de ar pela ferida, rouquidão, disfagia, sintomas neurológicos e choque hipovolêmico) e hora de admissão dos pacientes.

Num segundo momento, relacionaram-se os pacientes com ferimento penetrante. Através da análise dos prontuários médicos referentes a estes, relacionaram-se e analisaram-se os que receberam tratamento cirúrgico, os que receberam exames complementares e os que se mantiveram sob observação clínica.

Tabela 1 - Mecanismo de trauma.

\begin{tabular}{lrr}
\hline Mecanismo & $\mathbf{n}$ & $\boldsymbol{\%}$ \\
\hline FAF & 90 & $47 \%$ \\
FAB & 88 & $46 \%$ \\
Mordedura de Cão & 8 & $4 \%$ \\
Outros & 5 & $3 \%$ \\
\hline Total & 191 & $100 \%$ \\
\hline
\end{tabular}

Dos pacientes que foram submetidos a procedimento cirúrgico, observamos as lesões encontradas; os que foram encaminhados aos exames complementares, especificaram-se os exames empregados.

Empregou-se o teste Exato de Fisher para análise estatística, considerando-se o nível de significância de $5 \%(\mathrm{P}<0,05)$. Foram também aplicados aos resultados os limites de confiança de $95 \%$.

\section{RESULTADOS}

Cento e sessenta e quatro (86\%) pacientes eram do sexo masculino [limite de $95 \%$ de confiança para esta proporção é $(0,8092 ; 0,9080)]$ e $27(14 \%)$ do feminino. A média de idade foi de 28 anos (10 72). Dos 191 pacientes, 90 (47\%) foram vítimas de ferimentos por arma de fogo [limite de $95 \%$ de confiança: $(0,4004 ; 0,5420)], 88(46 \%)$ por armas brancas, oito $(4 \%)$ por mordeduras de cães e cinco (3\%) por outras causas (tabela 1). O horário em que se admitiu um maior número de pacientes foi entre $20 \mathrm{e}$ 04 horas (gráfico1).

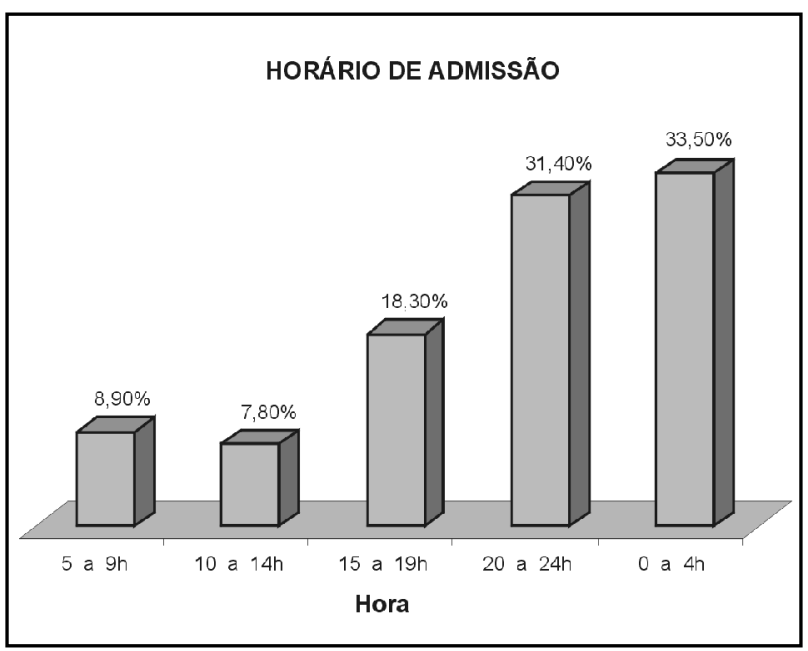

Gráfico 1 - Horário de admissão dos pacientes.

Em relação ao local da lesão, em 142 pacientes foi indicado o hemídio da ferida, em 116 o trígono e em 73 a zona acometida (tabela 2).

Em 101 pacientes (53\%) o ferimento ultrapassou o músculo platisma (ferimento penetrante de pescoço). O limite de $95 \%$ de confiança para esta proporção é $(0,4580 ; 0,5996)$. Dentre estes, 92 foram internados e nove foram liberados após observação 
Tabela 2 - Localização dos Ferimentos.

\begin{tabular}{lcrrrrlll}
\hline Hemídio & $\mathbf{n}(\mathbf{1 4 2})$ & $\boldsymbol{\%}$ & Zona & $\mathbf{n ( 7 3 )}$ & $\boldsymbol{\%}$ & Trígono & $\mathbf{n ( 1 0 6 )}$ & $\boldsymbol{\%}$ \\
\hline $\mathrm{E}$ & 75 & 53 & I & 26 & 36 & Anterior & 46 & 43 \\
$\mathrm{D}$ & 64 & 45 & II & 40 & 55 & Posterior & 37 & 35 \\
M & 3 & 2 & III & 7 & 9 & Região Posterior & 23 & 22 \\
\hline
\end{tabular}

Tabela 3 - Sinais e sintomas sugestivos de lesão (em número absoluto de sinais e sintomas).

\begin{tabular}{ccccccccc}
\hline Sinais e Sintomas S.A & TRM & ES & LPB & Disfagia & Fuga & Aérea Hemoptise Total \\
\hline$n$ & 16 & 14 & 12 & 5 & 3 & 3 & 3 & 2 \\
\hline
\end{tabular}

S.A.: sangramento ativo; TRM: trauma raquimedular; E. S.: Enfisema Subcutâneo; LPB: lesão de plexo braquial

de 12 horas (sem sinais e sintomas clínicos). Vinte e três ferimentos foram transfixantes.

Dentre os 92 pacientes internados, $51(55 \%)$ apresentaram sinais e sintomas clínicos significativos sugestivos de lesão: 16 (8\%) demonstravam sangramento ativo pela ferida, dois hemoptise, três disfagia, três rouquidão e em outros três observouse escape de ar pela ferida. Doze $(6 \%)$ foram os pacientes com enfisema subcutâneo. Quatorze (7\%) pacientes apresentaram clínica de traumatismo raquimedular e cinco, lesão de plexo braquial (tabela 3$)$.

Dos 101 pacientes com ferimento penetrante, $51(50 \%)$ receberam conduta operatória [limite de $95 \%$ de confiança: $(0,4075 ; 0,6025)]$, dos quais 32 (63\%) foram vítimas de ferimentos por arma de fogo, $18(18 \%)$ por arma branca e um $(1 \%)$ por mordedura de cão. Apesar dos pacientes vítimas de arma de fogo terem sido a maioria dos operados, proporcionalmente, em virtude do menor número de instrumentos "não FAF" terem penetrado o platisma, estes foram os que mais levaram à indicação de tratamento operatório. (tabela 4) $(\mathrm{p}=0,0136)$.

Dentre os 51 pacientes operados, a zona II, com 29 pacientes $(57 \%)$, foi a mais acometida [limite de $95 \%$ de confiança: $(0,4327 ; 0,7045)]$. Seguiu-se a zona I com 20 (39\%) e a zona III com dois $(4 \%)$. O trígono anterior (28 pacientes, ou seja, $55 \%$ ), foi o que mais indicou a terapêutica operatória [limite de $95 \%$ de confiança: $(0,4124 ; 0,6856)]$. Foram operados 14 (27\%) pacientes com feridas em trígono posterior e nove $(18 \%)$ em região posterior do pescoço (tabela 5).
Quando analisamos os procedimentos cirúrgicos relacionados aos exames complementares, observou-se que: dos 40 pacientes com lesão em zona II, dez (25\%) foram transfixantes com sinais e sintomas, acometendo também as zonas I, III e II contralateral. Em 12 (30\%) não havia sinais e sintomas clínicos significantes, sendo que em um caso o ferimento cruzava a linha média. Dos 26 ferimentos de zona I, em 10 (38\%) não havia sinais e sintomas. Dentre estes 10 , em quatro a indicação cirúrgica deveu-se aos exames complementares. Já nas lesões de zona III sem sinais e sintomas, em $28,57 \%$ os exames complementares indicaram a terapêutica cirúrgica.

As lesões encontradas são demonstradas na tabela 6. A cervicotomia foi não terapêutica em $47 \%$ dos casos.

Dos 92 pacientes internados, em 41 (45\%) o tratamento foi conservador de acordo com os sinais e sintomas clínicos e com os resultados dos exames complementares de endoscopia respiratória, digestiva e investigação por eco-Doppler em pacientes com sinais sugestivos de lesão vascular. Os pacientes com

Tabela 4 - Relação entre mecanismo de trauma e indicação de cirurgia em ferimentos penetrantes $(P=0,0136)$.

\begin{tabular}{|c|c|c|}
\hline CIRURGIA & FAF & NÃO FAF \\
\hline SIM & $32(41,56 \%)$ & $19 \quad(79,17 \%)$ \\
\hline NÃO & $45 \quad(58,44 \%)$ & $5 \quad(20,83 \%)$ \\
\hline Total & $77(100 \%)$ & $24(100 \%)$ \\
\hline
\end{tabular}


Tabela 5 - Local dos ferimentos nos pacientes submetidos à conduta operatória.

\begin{tabular}{lcclcc}
\hline Zona & $\mathbf{n}(\mathbf{5 1})$ & $\mathbf{\%}$ & Trígono & $\mathbf{n}(\mathbf{5 1})$ & \% \\
\hline Zona I & 20 & 39 & Anterior & 28 & 55 \\
Zona II & 29 & 57 & Posterior & 14 & 27 \\
Zona III & 2 & 4 & Região Posterior & 9 & 18 \\
\hline
\end{tabular}

Tabela 6 - Número e tipo das lesões.

\begin{tabular}{c|ccccccc}
\hline Lesões & Vasculares & Neurológicas & Aéreas & Digestivas & Outros & Total de Lesões & Total de Pacientes \\
\hline$n$ & 20 & 19 & 15 & 4 & 6 & 58 & 51 \\
\hline
\end{tabular}

feridas por armas de fogo foram sempre conduzidos com exames radiológicos. Foram realizadas 17 broncoscopias, 15 endoscopias digestivas altas e nove exames vasculares por eco-Doppler. Apenas seis (14\%) destes pacientes receberam tratamento operatório de acordo com os exames complementares, sendo que quatro foram lesionados em zona I e dois em zona III.

\section{DISCUSSÃO}

Os ferimentos que acometem a região cervical, por sua complexidade e morbidade, certamente merecem atenção dos serviços de emergência que prestam serviços à população. Obtivemos em nossa casuística uma análise bastante extensa dos principais elementos de interesse clínico e epidemiológico em relação às feridas de pescoço. Demonstramos assim o tipo de população vítima destas lesões, bem como o seu mecanismo, local, características clínicas e a conduta terapêutica empreendida.

O sexo masculino foi o mais acometido com $86 \%$ dos casos, achado que apenas confirmou o verificado na literatura, a qual apresenta valores entre $88 \%$ e $92 \%$. $^{3,5-8}$ Isso reflete uma maior exposição às situações de violência pelos homens. O mesmo observou-se quanto à idade ( 28 anos) que coincide com a faixa etária do adulto jovem encontrada na literatura. $^{3,5-10}$

Quanto ao mecanismo de trauma pode-se notar que este varia em relação à época e ao centro de trauma em que se elaborou a pesquisa. Nos tempos atuais e em países em que não há controle efetivo sobre o porte e uso de armas, os ferimentos por arma de fogo (FAF) predominam. Estes números variam entre $51 \%$ e $74 \%$ 5,6,8,9,23. Poucos autores encontraram um maior número de feridas por arma branca $(\mathrm{FAB})^{3,7,11,12,20,21}$. Nestes casos a porcentagem oscilou entre $52 \%$ e $95 \%$. Em nosso estudo praticamente não houve predomínio de um mecanismo sobre o outro, uma vez que encontramos $47 \%$ de FAFs e $46 \%$ de FABs. Quando avaliados os ferimentos penetrantes, os valores foram $77 \%$ FAF e $23 \%$ FAB.

$\mathrm{O}$ intervalo de horário onde houve um maior número de admissões foi entre $20 \mathrm{~h}$ e $04 \mathrm{~h}$, o que demonstra ser à noite e durante a madrugada que ocorre a maioria das lesões por violência urbana. Poucos trabalhos documentam esse dado em relação aos ferimentos cervicais.

Quanto à localização das lesões, verificamos uma maior incidência do lado esquerdo (53\%), o mesmo observado por Biffl et $\mathrm{al}^{8}$. A literatura nos mostra um maior acometimento da zona II, com $47 \%$ a $82 \%$, seguida da zona I, com $5 \%$ a $31 \%$ dos casos e da zona III com trabalhos relatando acometimentos em $1 \%$ a $30 \% .^{5-9,12-15}$ Com resultado semelhante, observamos em nosso estudo a freqüência de acometimento das zonas cervicais na mesma seqüência: $55 \%$ zona II, $36 \%$ zona I e $9 \%$ zona III. O trígono anterior foi o mais acometido (43\%). Isso tem sua importância uma vez que alguns autores como Walsh et $\mathrm{al}^{23}$. advogam a exploração mandatória quando do acometimento do trígono anterior.

Existem ainda controvérsias entre os autores quanto ao tratamento operatório ou conservador das lesões penetrantes de pescoço. Alguns sugeriram empiricamente que a conduta operatória seria a adequada para lesões por arma de fogo e conservadora 
para feridas por arma branca ${ }^{19}$. No entanto, apesar dos projetis de arma de fogo causarem mais lesões vasculares, aerodigestivas e medulares, existe uma incidência significativa de sinais sugestivos de lesões vasculares nos ferimentos por arma branca. Há estudos que demonstram que a necessidade dos procedimentos terapêuticos ocorre em aproximadamente 16\% nas lesões penetrantes por arma de fogo e $10 \%$ nas por arma branca. ${ }^{5}$ Quando a conduta operatória é empregada de rotina são observadas taxas elevadas de cirurgias desnecessárias. ${ }^{5,21}$

Dos 101 pacientes em que o ferimento ultrapassou o platisma, 92 foram internados. O internamento teve por objetivo o tratamento cirúrgico, a observação clínica mais detalhada ou a execução de exames complementares ${ }^{25}$. Por apresentarem sinais e sintomas significativos sugestivos de lesão de estruturas nobres, 51(50\%) pacientes foram operados, na maioria vítimas de FAF. Este percentual corresponde ao que relatam alguns autores ${ }^{7,8,21}$. Ayuyao et $\mathrm{al}^{21}$. ao utilizarem-se do manejo seletivo, exploraram $40(37 \%)$ de 109 pacientes.

Das 51 explorações realizadas em nossa casuística, 24 (47\%) foram não terapêuticas. Um número elevado quando relacionado aos achados da literatura. Biffl et $\mathrm{al}^{8}$. realizaram $16 \%$ de explorações negativas e Irish et $\mathrm{al}^{7}, 14 \%$. No entanto, casuísticas que pregam o uso de exploração mandatória para todos os pacientes com feridas penetrantes de pescoço revelam taxas elevadas de explorações não terapêuticas ${ }^{11,26}$, a exemplo de Apffelstaedt et $\mathrm{al}^{22}$, que realizou 226 (57\%) explorações negativas dentre 393 pacientes durante 1,8 ano. Observa-se neste caso a necessidade de se explorar todos os ferimentos quando o serviço recebe um número muito elevado de doentes num curto período.

As lesões vasculares foram os achados mais freqüentes nas cervicotomias terapêuticas, seguidas das lesões neurológicas, das vias aéreas e das lesões do aparelho digestório. Estes valores se assemelham aos encontrados na literatura, que seguem esta mesma ordem de frequiência ${ }^{5,15-18}$.

Existe grande discussão em torno da conduta a ser adotada em relação à zona acometida. Alguns autores advogam o empreendimento dos exames complementares de rotina para pacientes com feridas em zonas I e III devido à dificuldade da exposição cirúrgicas nestes locais. ${ }^{20}$ Já outros acreditam que independentemente do local da ferida, a angiografia e endoscopia de rotina devem ser empregadas sempre que nas proximidades do esôfago e estruturas vasculares..$^{27,28}$ Esta preocupação se deve à tentativa de se evitar operações não terapêuticas, que ocorrem em larga escala quando do uso de condutas cirúrgicas mandatórias. ${ }^{11,20-23}$. Ainda existe grande controvérsia em relação à zona II quanto à exploração mandatória ou seletiva. Segundo Asensio ${ }^{15,24}$, ambas se equivalem, pois uma exploração indispensável é segura e aceitável, enquanto a abordagem seletiva com angiografia nos assintomáticos também é muito eficaz.

Em nosso estudo observamos que a zona II é a que mais indica o tratamento operatório (57\%). Anatomicamente, a maioria das feridas é em zona II. 5-9,11-14 Esta situação se repetiu no estudo de Biffl et $\mathrm{al}^{8}$, onde a zona II (38\%) foi a que mais indicou cirurgia.

O tratamento conservador, com a realização de exames complementares e de internamento dos pacientes, foi adotado em $40 \%$ dos 101 pacientes cujo ferimento penetrou o platisma. A maioria destes pacientes estavam hígidos ou apresentaram lesões que não necessitaram correção cirúrgica. $\mathrm{O}$ valor corresponde ao observado em alguns trabalhos. ${ }^{6,21}$ Irish et $\mathrm{al}^{7}$, realizaram tratamento conservador em $30 \%$ dos pacientes, e Ayuyao et $\mathrm{al}^{21}$, em $63 \%$.

Por fim, acreditamos que os ferimentos cervicais são melhor tratados com a conduta seletiva. O procedimento cirúrgico mandatório "de princípio" deve ser substituído pela operação imediata somente quando as condições clínicas do paciente assim o exigirem. A sistematização diagnóstica deve compreender a radiografia simples da coluna cervical, o esofagograma + esofagoscopia e a nasofaringolarinoscopia nos casos com sinais clínicos de lesão ou ferimentos transfixantes. Reserva-se a traqueobroncoscopia para os casos onde há suspeita de lesão de via aérea e se a nasofaringolaringoscopia for inconclusiva. O ecoDoppler de carótidas e vertebrais deve ser feito em todo ferimento das zonas I e III . Os casos suspeitos devem ser confirmados pela angiotomografia. Quanto à zona II, o eco-Doppler deve der indicado baseando-se no exame clínico.

Conclui-se que o indivíduo jovem do sexo masculino é o mais acometido em lesões cervicais, há relativa equivalência quanto ao mecanismo de trauma e a zona II e o trígono anterior são os locais de maior incidência de ferimentos e operações. $O$ fato 
do número de cervicotomias não terapêuticas ter sido elevado mostrou haver a necessidade de um manejo mais seletivo em relação aos ferimentos cervicais, devendo o manejo da zona II adequar-se aos dados epidemiológicos e à disposição de recursos dos serviços de trauma.

\begin{abstract}
Background: The study proposes to assess the demographics and management of neck injuries. Methods: A retrospective review of 487.128 emergency department medical records from 01/1996 to 06/2001 was conducted. Out of the 378 patients selected for the analysis, 153 were excluded due to associated lesions and 14 because of death. The group of patients analyzed $(n=191)$ had exclusively neck lesions. Injury location, kinetics of trauma, platysma involvement, signs and symptoms, admission hour and management were evaluated. Results: One hundred sixty four (86\%) patients were male. Mean age was 28 years (range, 10-72). Ninety (47\%) injuries were caused by firearms and 88 (46\%) were stab wounds. The main period of admission was between $08 \mathrm{pm}$ and $04 \mathrm{am}$. The location of the lesions was 53\% in the left, $45 \%$ in the right, and $2 \%$ in the median line; $36 \%$ of them being classified as zone I, 55\% as II, and $9 \%$ as III. There was platysma penetration in 101 patients, half of them $(n=51)$ presented signs and symptoms suggestive of injury, and were consequently subjected to surgical intervention. Twenty-four (47\%) operative interventions were non-therapeutic. Forty-one patients (45\%) were medically treated on the basis of clinical assessment. Conclusions: Young males are the most frequent victims of neck injuries. It occurs mostly in the Zone II, and the incidence of firearm and stab wounds were equivalent as a trauma mechanism. The necessity of a selective management of neck lesions is clearly evident. Regarding penetrating zone II neck injuries, the treatment may be adequated with the resources of trauma services.
\end{abstract}

Key Words: Neck injuries; Epidemiology; Analysis; Therapeutical approaches.

\section{REFERÊNCIAS}

1. Mattox KL, Feliciano DV, Moore EE - Trauma. $4^{\mathrm{a}}$ Edition. New York - McGraw-Hill, 2000.

2. Gardner E, Gray DJ, O'Rahhily R - Anatomia. 4ª Edição. Rio de Janeiro - Guanabara- Koogan, 1988, pp. 670-673.

3. Lourenção JL, Nahas SC, Margarido NF, et al. Ferimentos penetrantes cervicais: análise prospectiva de 53 casos. Rev Hosp Clin Fac Med São Paulo,1998, 53(5): 234-241.

4. American College of Surgeons Committee on Trauma: Advanced Trauma Life Support Course. Chicago, American College of Surgeons, 1998.

5. Demetríades D, Theodorou D, Cornwell E, et al. Evaluation of penetrating injuries of the neck : prospective study of 223 patients. World J Surg, 1997, 21(1): 41-48.

6. Meyer JP, Barrett JA, Schuler JJ, et al. - Mandatory vs selective exploration for penetrating neck trauma. A prospective assessment. Arch Surg,1987, 122(5): 592-597.

7. Irish JC, Hekkenberg R, Gullane PJ, et al. - Penetrating and blunt neck trauma: 10-year review of a Canadian experience. Can J Surg, 1997, 40(1):33-38.
8. Biffl WL, Moore EE, Rehse DH, et al. - Selective management of penetrating neck trauma based on cervical level of injury. Am J Surg,1997, 174(6):678682.

9. Demetriades D, Theodorou D, Cornwell E, et al. Penetrating injuries of the neck in patients in stable condition. Physical examination, angiography, or color flow Doppler imaging. Arch Surg, 1995, 130(9):971-975.

10. Flowers JL, Graham SM, Ugarte MA, et al. - Flexibe endoscopy for the diagnosis of esophageal trauma. J Trauma, 1996, 40(2):261-265.

11. Nason RW, Assuras GN, Gray PR, et al. - Penetrating neck injuries: analysis of experience from a Canadian trauma centre. Can J Sug, 2001, 44(2):122-126.

12. Atta HM, Walker ML - Penetrating neck trauma: lack of universal reporting guidelines. Am Surg, 1998, 64(3):222-225.

13. Klyachkin ML, Rohmiller M, Charash WE, et al. Penetrating injuries of the neck: selective management evolving. Am Surg, 1997, 63(2):189-194.

14. Wood J, Fabian TC, Mangiante EC - Penetrating neck injuries: recommendations for selective management. J Trauma, 1989, 29(5):602-605. 
15. Demetríades D, Asensio J, Velmahos G - "Problemas complexos no traumatismo cervical penetrante". In Asensio J, Demetriades D, Berne T (eds) - Clínicas Cirúrgicas da América do Norte. Problemas complexos e desafiadores na cirurgia traumatológica. Rio de Janeiro. Interlivros, 1996, vol. 4, pp. 681-682.

16. McConnell DB, Trunkey DD - Management of penetrating trauma to the neck. Adv Surg, 1994, 27:97127.

17. Demetriades D, Charalambides D, Lakhoo M - Physical examination and selective conservative management in patients with penetrating injuries of the neck. Br J Surg,1993, 80(12):1534-1536.

18. Pate JW - Tracheobronchial and esophageal injuries. Surg Clin North Am, 1989, 69(1):111-123.

19. Shenk WG - Neck Injuries. In Moylan JA (ed) Principles of trauma surgery. New York. Gower Mediacol Publisher,1992, pp. 1510-1515.

20. Bishara RA, Pasch AR, Douglas DD, et al.- The necessity of mandatory exploration of penetrating zone II neck injuries. Surgery, 1986,100(4): 655-660.

21. Ayuyao AM, Kaledzi YL, Parsa MH, et al. - Penetrating neck wounds. Mandatory versus selective exploration. Ann Surg, 1985, 202(5):563-567.

22. Apffelstaedt JP, Muller R - Results of mandatory exploration for penetrating neck trauma. World J Surg, 1994, 18(6):917-919.
23. Walsh MS - The management of penetrating injuries of the anterior triangle of the neck injury. 1994, 25(6):393395.

24. Asensio JA, Valenziano CP, Falcone RE, et al. Management of penetrating neck injuries. The controversy surrounding zone II injuries. Surg Clin North Am,1991, 71(2):267-296.

25. Ginzburg E, Montalvo B, LeBlang S, et al. - The use of duplex ultrasonography in penetrating neck trauma. Arch Surg, 1996, 131(7):691-693.

26. Gerst PH, Sharma SK, Sharma PK - Selective management of penetrating neck trauma. Am Surg,1990, 56(9):553-555.

27. Sclafani SJ, Cavaliere G, Atweh N, et al. - The role angiography in penetrating neck trauma. J Trauma,1991,31(4):557-562.

28. Weigelt JA, Thal ER, Snyder WH, et al. - Diagnosis of penetrating cervical esophageal injuries. Am J Surg,1987, 154(6):619-622.

Endereço para correspondência:

Dr. Luiz Carlos Von Bahten

Endereço: Rua Martin Afonso 2642 ap.1902

Bigorrilho

CEP: 80730-030 Curitiba-PR

E-mail: bahten@netpar.com.br 